\title{
SRG/ART-XC discovery of SRGA J204318.2+443815: towards the complete population of faint X-ray pulsars
}

\author{
A.A. Lutovinov ${ }^{\star 1}$, S.S. Tsygankov ${ }^{1,2}$, I.A. Mereminskiy ${ }^{1}$, S.V. Molkov ${ }^{1}$, A.N. Semena ${ }^{1}$, V.A. Arefiev ${ }^{1}$, I.F. Bikmaev ${ }^{3}$, \\ A.A. Djupvik ${ }^{4,5}$, M.R. Gilfanov ${ }^{1,6}$, D.I. Karasev ${ }^{1}$, I.Yu. Lapshov ${ }^{1}$, P.S. Medvedev ${ }^{1}$, A.E. Shtykovsky ${ }^{1}$, R.A. \\ Sunyaev $^{1,6}$, A.Yu. Tkachenko ${ }^{1}$, S. Anand ${ }^{7}$, M.C.B. Ashley ${ }^{8}$, K. De ${ }^{9}$, M.M. Kasliwal ${ }^{7}$, S.R. Kulkarni ${ }^{7}$, J. van Roestel ${ }^{7}$, \\ and $\mathrm{Y} . \mathrm{Yao}^{7}$
}

\begin{abstract}
1 Space Research Institute (IKI) of Russian Academy of Sciences, Prosoyuznaya ul 84/32, 117997 Moscow, Russian Federation 2 Department of Physics and Astronomy, FI-20014 University of Turku, Finland

3 Kazan Federal University, Kremlevskaya Str., 18, Kazan, Russian Federation

4 Nordic Optical Telescope, Apartado 474, 38700 Santa Cruz de La Palma, Santa Cruz de Tenerife, Spain

5 Department of Physics and Astronomy, Aarhus University, NyMunkegade 120, DK-8000 Aarhus C, Denmark

${ }^{6}$ Max Planck Institute for Astrophysics, Karl-Schwarzschild-Str. 1, Postfach 1317, D-85741 Garching, Germany

7 Division of Physics, Mathematics and Astronomy, California Institute of Technology, Pasadena, CA 91125, USA

8 School of Physics, University of New South Wales, Sydney NSW 2052, Australia

9 Cahill Center for Astrophysics, California Institute of Technology, Pasadena, CA 91125, USA
\end{abstract}

July 13,2021

\begin{abstract}
We report a discovery of a new long-period X-ray pulsar SRGA J204318.2+443815/SRGe J204319.0+443820 in the Be binary system. The source was found in the second all-sky survey by the Mikhail Pavlinsky ART-XC telescope on board the SRG mission. The follow-up observations with XMM-Newton, NICER and NUSTAR observatories allowed us to discover a strong coherent signal in the source light curve with the period of $\sim 742 \mathrm{~s}$. The pulsed fraction was found to depend on the energy increasing from $\sim 20 \%$ in soft X-rays to $>50 \%$ at high energies, as it is typical for X-ray pulsars. The source demonstrate a quite hard spectrum with an exponential cutoff at high energies and bolometric luminosity of $L_{X} \simeq 4 \times 10^{35} \mathrm{erg} \mathrm{s}^{-1}$. Dedicated optical and infrared observations with the RTT-150, NOT, Keck and Palomar telescopes revealed a number of emission lines $\left(\mathrm{H}_{\alpha}, \mathrm{He}\right.$, Pashen and Braket series) with the strongly absorbed continuum. All of above suggests that SRGA J204318.2+443815/SRGe J204319.0+443820 is a new persistent low luminosity X-ray pulsar in a distant binary system with a Be-star of the B0-B2e class. Thus the SRG observatory allow us to unveil the hidden population of faint persistent objects including the population of slowly rotating X-ray pulsars in Be systems.
\end{abstract}

Key words. pulsars: individual: (SRGA J204318.2+443815) - stars: neutron - X-rays: binaries

\section{Introduction}

The key task of Spectrum Roentgen Gamma (SRG) mission (Sunyaev et al. 2021) is the deepest all-sky survey in X-rays, both at soft energies $0.3-10 \mathrm{keV}$ with the eROSITA telescope (Predehl et al.2021) and the hard ones $4-30 \mathrm{keV}$ with the Mikhail Pavlinsky ART-XC telescope (Pavlinsky et al. 2021). Surveying about $1 \%$ of the sky daily, down to the fluxes of $\simeq(1-2) \times 10^{-11}$ erg $\mathrm{cm}^{-2} \mathrm{~s}^{-1}$ in the $4-12 \mathrm{keV}$ band (about 1-1.5 mCrab), the ART-XC telescope is providing a unique possibility to study the population of faint transients that would be otherwise missed, being too weak for the wide field-of-view telescopes and all-sky monitors (like INTEGRAL/IBIS, Swift/BAT, MAXI). One of the most intriguing families of such a population are accreting neutron stars with very strong magnetic fields (X-ray pulsars) allowing studies of an interaction of the matter with the magnetic field at very low mass accretion rates.

On Nov 20, 2020 during the second consecutive all-sky survey, the Mikhail Pavlinsky ART-XC telescope discovered a relatively bright $\mathrm{X}$-ray source with coordinates of $\mathrm{RA}=310.8259$, Dec $=44.6374(\mathrm{~J} 2000)$ and the flux of $\sim 2.4 \times 10^{-11} \mathrm{erg} \mathrm{s}^{-1} \mathrm{~cm}^{-2}$

^aal@iki.rssi.ru in the 4-12 keV energy band (Mereminskiy et al. 2020). The source was also detected by eROSITA that allowed to obtain an enhanced position of the source $(\mathrm{RA}=310.8293$, $\mathrm{Dec}=44.6390$, $\mathrm{J} 2000$, 95\% confidence radius is $4^{\prime \prime}$ ) and to perform a preliminary spectral analysis in soft X-rays (Mereminskiy et al. 2020). The eROSITA position is found to be consistent with the optical transient ZTF18abjpmzf in turn identified with a distant star at $\sim 8.0_{-1.9}^{+2.8} \mathrm{kpc}$ using the Gaia survey (Bailer-Jones et al. 2021). This distance value we will use in the following calculations.

The source ZTF18abjpmzf has been monitored by the Zwicky Transient Facility (ZTF; Bellm et al. 2019, Graham et al. 2019) since March 2018. The median of all ZTF detection positions is $($ R.A., Dec $)=(310.827745,44.638897)=$ (20h43m18.66s, +44d38m20.0s) (J2000), that corresponds to the Galactic coordinates $(l, b)=(83.9837,1.3407)$. The optical source is coincident with a bright infrared star detected in 2MASS (Skrutskie et al. 2006). This star exhibited a variable infrared emission in the $J$-band based on data taken with the Palomar Gattini-IR survey (PGIR; Moore \& Kasliwal 2019, De et al. 2020c) since November 2018.

Follow-up optical observations as well as an inspection of the archival data revealed a strong $\mathrm{H} \alpha$ line in the optical spectrum and optical/IR variability (Yao et al.2020; De et al.|2020b). 
Given these measurements as well as a low Galactic latitude of the transient and strong absorption in its spectrum we could assume that SRGA J204318.2+443815 is a new high-mass X-ray binary (HMXB) system, harboring a neutron star and a Be companion.

In this paper we report a discovery of a new transient SRGA J204318.2+443815 and results of its follow-up observations in X-rays with XMM-Newton, NuSTAR, Swift/XRT and NICER and optics with the RTT-150, NOT, Keck and Palomar telescopes. These observations allow to discover coherent pulsations with the period of $\sim 742 \mathrm{~s}$ in the source light curve, measure its broadband spectrum and reveal a Be-nature of the optical counterpart.

\section{Observations and data analysis}

\subsection{X-ray observations}

The Spectrum Roentgen Gamma (SRG) mission is a project of the Russian Federal Space program that hosts two X-ray telescopes: the Mikhail Pavlinsky ART-XC and eROSITA. The Mikhail Pavlinsky ART-XC telescope is a grazing incidence focusing X-ray telescope which provides imaging, timing and spectroscopy in the 4-30 keV energy range (Pavlinsky et al. 2021). The telescope consists of seven identical modules with the total effective area of $\sim 450 \mathrm{~cm}^{2}$ at $6 \mathrm{keV}$, angular resolution of $\sim 50^{\prime \prime}$, energy resolution of $1.4 \mathrm{keV}$ at $6 \mathrm{keV}$ and timing resolution of $23 \mu \mathrm{s}$. ART-XC data were processed with the analysis software ARTPRODUCTS v0.9 with the CALDB version 20200401. During the second SRG survey SRGA J204318.2+443815 was observed with a total exposure of $125 \mathrm{~s}$ (an effective exposure time of $\sim 37 \mathrm{~s}$ ). Taking into account the faintness of the source, short exposure and limited number of photons we attributed all counts detected by ART-XC into two broad energy bins (4-12 an 12-26 keV) and modeled the ART-XC data jointly with the contemporary eROSITA spectrum. eROSITA detected the source SRGe J204319.0+443820 in both surveys: at the moment of its discovery with ART-XC in eRASS2 (Nov 18-21, 2020) with a total exposure of $252 \mathrm{~s}$ and (based on the retrospective analysis) in eRASS1 (May 18-22, 2020), where it was observed with a total exposure of 289 s. eROSITA data were processed with the eROSITA pipeline at IKI based on eSASS package components and data analysis software developed at IKI. SRGA J204318.2+443815/SRGe J204319.0+443820 was also significantly detected by ART-XC and eROSITA during third survey on May 21, 2021 with the total exposures of 138 and $227 \mathrm{~s}$, respectively.

Soon after the detection of the source we initiated NICER follow-up observations in order to examine its short-scale variability. Due to different reasons no observations were carried out until Dec 04, 2020, when first short (900 s) observation was performed. Later, more longer observation was carried out on Dec 11, 2020, which lasted for $8 \mathrm{ks}$. We used a standard NICERDAS in order to extract spectra and light curves. Unfortunately, the source was too obscured and weak, with energies below $\approx 2$ $\mathrm{keV}$ and above $\approx 5 \mathrm{keV}$ being dominated by a non-photon background. Therefore we decided to not use spectral data. In the 2-6 keV energy band we found a significant modulation with the period of $741.8 \mathrm{~s}$, which shape is roughly consistent with measurements by XMM-Newton.

To improve further the source localization and to study in depth its spectral an timing properties in soft X-rays, we asked follow-up DDT observations of SRGA J204318.2+443815 with the XMM-Newton observatory. Observations were performed on
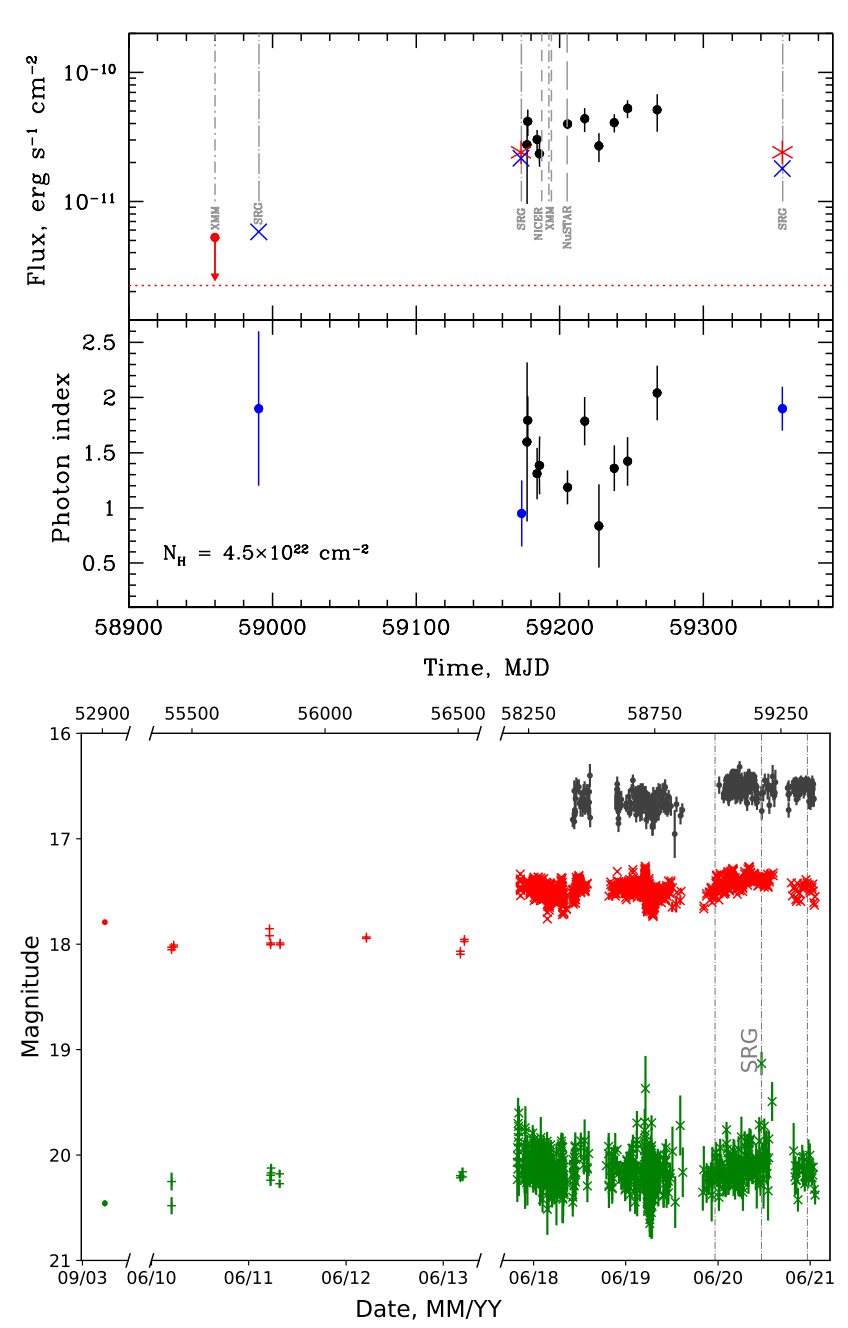

Fig. 1. Top panel. Light curve of SRGA J204318.2+443815 obtained with $S$ wift/XRT (black points), eROSITA (blue crosses) in the $0.5-10$ $\mathrm{keV}$ band and ART-XC (red crosses) in the 4-12 keV band. Middle panel shows a corresponding dependence of the photon index assuming a simple absorbed power law spectral model with $N_{\mathrm{H}}$ fixed at the value of $4.5 \times 10^{22} \mathrm{~cm}^{-2}$, determined from the average spectrum. Times of the source observations with other X-ray instruments are shown with vertical grey lines: SRG (long dashed-dotted), NICER (dashed), XMMNewton (dashed-dotted), NuSTAR (long dashed). The source flux measured by ART-XC and eROSITA during the discovery and in the first and third surveys are shown with the red asterisk and blue crosses, respectively. Upper limits for the source flux from the XMM-Newton slew surveys in Apr 2020 and Dec 2011 are shown by the red circle and red dotted line, respectively. Bottom panel: long-term optical light curve of ZTF18abjpmzf in $g(r)$ filters shown in green (red). Earliest measurements (dots) are from SDSS, PanSTARRS data shown with pluses and ZTF with crosses. Also, $J$-filter measurements from PGIR (shifted for clarity by +4 magnitudes) is shown with black dots. Secular long-term brightening is clearly seen in $r$ filter measurements, along with irregular short-scale low-amplitude variability.

Dec 9, 2020 (ObsID 0872391201). To process the XMM-Newton data, we used version 18.0 of the XMM-Newton Science Analysis System (SAS). For the analysis of the EPIC data we selected events with patterns in the range $0-4$ for the pn camera and 0-12 for the two MOS cameras, using a circular region with a radius of $20^{\prime \prime}$ around the source position. Background events were selected from circular regions (with radius of $30^{\prime \prime}$ ) offset from the source position. 


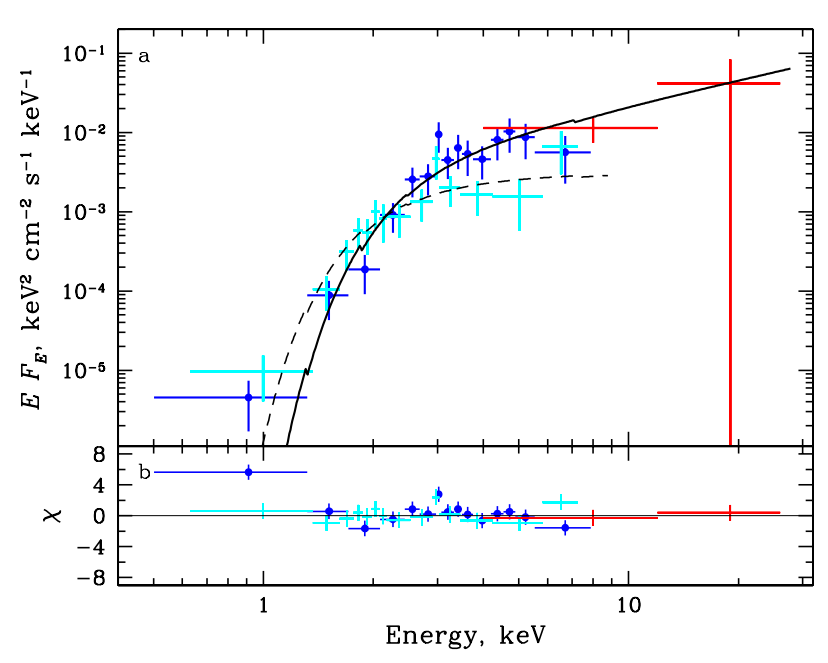

Fig. 2. Spectra of SRGA J204318.2+443815 measured with $S R G$ instruments. Blue and red points correspond to eROSITA and ART-XC observations in the second survey, respectively. Cyan points represent the spectrum obtained by eROSITA during the first survey. Solid and dashed lines are corresponding best-fit models.

To trace the long-term variability of the source observations with the X-ray telescope (XRT; Burrows et al. 2005) on-board the Neil Gehrels Swift Observatory (Gehrels et al. 2004) were triggered. This monitoring lasted for three months from November 24, 2020 to February 22, 2021. All XRT data were taken in the photon counting mode and reduced using the online tools ${ }^{1}$ (Evans et al. 2009) provided by the UK Swift Science Data Centre. The resulting spectra were fitted with a simple absorbed power law model in the $0.3-10 \mathrm{keV}$ band using the XsPEC package (Arnaud 1996).

Finally in our analysis we used the NUSTAR data to confirm detection of the pulsations, which were initially found in the XMM-Newton and NICER data, as well as to study properties of the source in the broad X-ray band. The NUSTAR observatory consists of two identical X-ray telescope modules, referred to as FPMA and FPMB (Harrison et al. 2013). It provides $\mathrm{X}$-ray imaging, spectroscopy and timing capabilities in the energy range of 3-79 $\mathrm{keV}$. NuSTAR performed an observation of SRGA J204318.2+443815 on Dec 22, 2020 (ObsID 90601338002). The data reduction for this observation was done using HEASOFT v6.28 and CalDB version 20210210. All spectra were binned to have at least one count per energy bin and W-statistid ${ }^{2}$ was applied (Wachter et al. 1979).

\subsection{Optical and infrared data}

RTT-150. Optical observations from the Russian-Turkish Telescope (RTT-150) have been performed by using the TFOSC instrument on 24-25 Nov, 2020 and 9 Apr, 2021 (low resolution spectrometer and imager) equipped with the Andor CCD (model DZ936, BEX2 DD chip) cooled to -80 C. We used g,r,i,z filters for photometrical observations. Note, that during RTT-150 observations the optical counterpart was by 0.3-0.5 mag brighter in comparison with SDSS DR16 archive data, showing long term variations its brightness between November 2020 and April

\footnotetext{
1 http://www.swift.ac.uk/user_objects/

2 https://heasarc.gstc.nasa.gov/xanadu/xspec/manual/ XSappendixStatistics.html
}

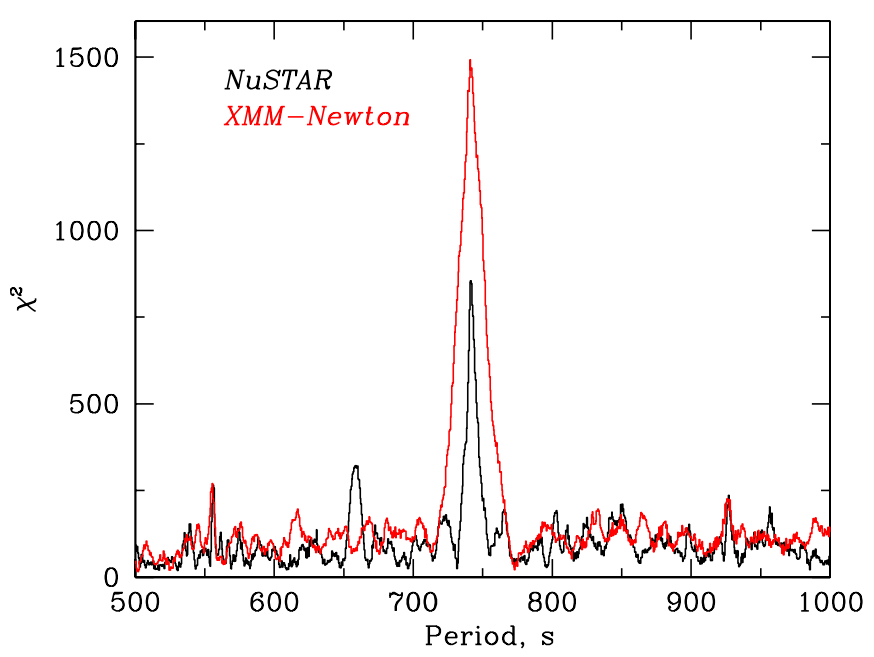

Fig. 3. Periodogram of the source, obtained by NUSTAR (black) and $X M M-N e w t o n$ (red). A coherent signal is clearly detected by both instruments around $\simeq 742 \mathrm{~s}$.

2021. At the same time we found no fast variations in brightness at the time scale of 3 hours during RTT-150 photometrical observations on Nov 24, 2020, when we have obtained two low resolution (15 Angstrom) spectra with the exposure of $900 \mathrm{sec}$ each.

NOT. At the $2.56 \mathrm{~m}$ Nordic Optical Telescope we applied for time through the fast-track access and obtained an optical lowresolution spectrum on 2021 March 18 with ALFOSC and nearIR low-resolution spectra on April 25 with NOTCam. The ALFOSC spectrum with Grism \#4 and a $1^{\prime \prime}$ wide slit covers the wavelength range 3200 to $9600 \AA$ with a resolving power of $\mathrm{R}=$ 360. The exposure time was 2700s. The near-IR spectra were obtained with NOTCam Grism \#1 and the broad-band filters $J$ ad $K$ as order sorters. The dispersions are 2.5 and $4.1 \AA /$ pix in J and $\mathrm{K}$, respectively, and with the $0.6^{\prime \prime}$ wide slit give a resolving power of $R=2100$. Six spectra were taken dithering the target along the slit with individual exposure times of $300 \mathrm{~s}$ using ramp-sampling readout modes. Each sky-subtracted and flat-fielded spectrum was optimally extracted and wavelength calibrated before combined to a final spectrum. A nearby bright star of spectral type A0 $\mathrm{V}$ was observed immediately before the target and used for telluric correction, removing primarily the stellar absorption lines, upon which a properly flux-scaled Vega model was multiplied back to restore the slope and obtain a rough flux calibration. For the $J$ band we used the telluric standard to find the conversion from $\mathrm{ADU} / \mathrm{s}$ to $\mathrm{f}_{\lambda}$, while for the $K$ band we used the target acquisition image with comparison stars.

Keck, Palomar. We obtained follow-up optical and nearinfrared spectra using the Low Resolution $(R \approx 1000)$ Imaging Spectrograph (LRIS/Keck-I; Oke et al. 1995) on 2020 December 12 , the medium-resolution $(R \approx 13000)$ Echellette Spectrograph and Imager (ESI/Keck-II; Sheinis et al.2002) on 2020 December 08 , and the medium-resolution $(R=2700)$ Triplespec spectrograph (Tspec/Palomar 200-inch telescope; Herter et al. 2008) on 2020 December 23.

\section{Source discovery and long term variability}

The long-term X-ray light curve covering about 3 months of observations of SRGA J204318.2+443815 with the Swift/XRT telescope after its discovery is presented in Fig. 1. As can be seen 

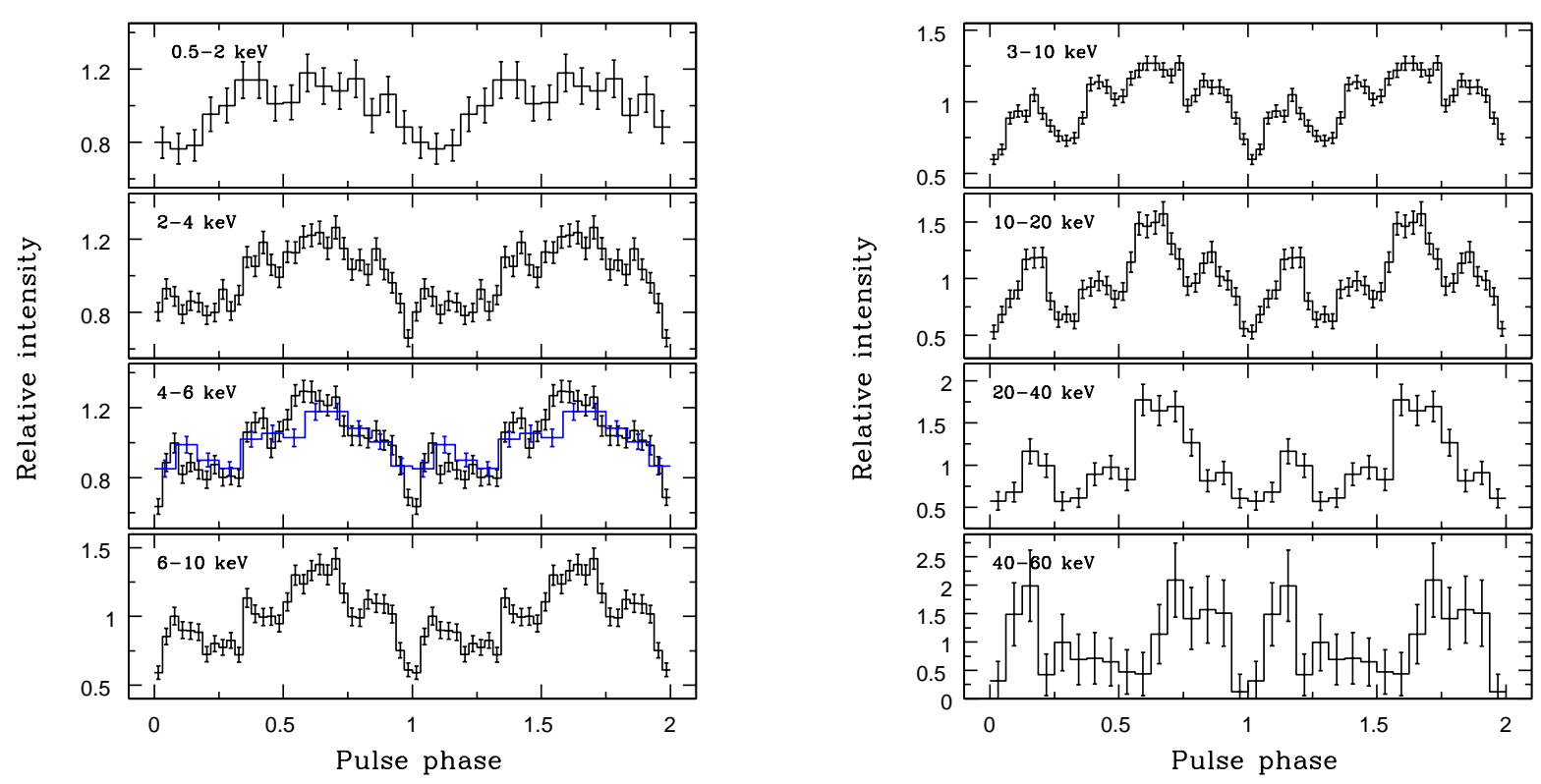

Fig. 4. Pulse profiles of SRGA J204318.2+443815 as a function of the energy as observed by XMM-Newton (left) and NuSTAR (right). Profile shown in blue colour represents results obtained from the NICER data in the 2-6 keV band. Zero phase in both datasets is chosen arbitrary to coincide with the main minimum in the profile.

from the figure, no significant source variability was revealed not only on this time scale, but also during a half of year after the discovery, when both $S R G$ instruments detected again SRGA J204318.2+443815 in May, 2021 approximately at the same level of intensity. At the same time the X-ray source exhibited a moderate variability between the first and the second $S R G$ all sky surveys separated by six months.

Additionally, an inspection of previous scans of this sky field during the XMM-Newton slew surveys 3 (Saxton et al. 2008) (in Jun, 2010, Dec, 2011, Dec, 2018 and Apr 2020) showed no detection of the source with the lowest flux limit of $2.2 \times$ $10^{-11} \mathrm{erg} \mathrm{s}^{-1} \mathrm{~cm}^{-2}$ in the $0.2-12 \mathrm{keV}$ energy band in 2011 (red dotted line in Fig. 11. This limit points to the source flux variability at least at the level of the order of magnitude.

As it was mentioned above the source SRGA J204318.2+443815 was initially discovered in the near-real time analysis of the ART-XC and eROSITA data during the second all-sky survey. The retrospective detailed analysis of the data from both instruments obtained during the first survey showed that the source was registered by eROSITA while staying below the detection threshold in the ART-XC data. The spectra of SRGA J204318.2+443815 measured by eROSITA + ART-XC and eROSITA in the first and second surveys, respectively, are shown in Fig. 2 . It is seen that both of them are quite similar and can be describe by an absorbed power law. The absorption values $N_{\mathrm{H}}=(3.0 \pm 0.8) \times 10^{22}$ and $N_{\mathrm{H}}=(4.5 \pm 0.8) \times 10^{22} \mathrm{~cm}^{-2}$, measured in first and second surveys, respectively, are compatible with each other and exceed the Galactic value in the source direction. It can indicate presence of the intrinsic absorption in the system similar to already found in a number of faint BeXRB systems. The source flux in the $0.5-10 \mathrm{keV}$ band obtained during the second survey $(2.2 \pm 0.4) \times 10^{-11} \mathrm{erg} \mathrm{s}^{-1} \mathrm{~cm}^{-2}$ increased by factor of $\simeq 3.7$ in comparison to the one in the first survey $5.8_{-1.6}^{+2.3} \times 10^{-12} \mathrm{erg} \mathrm{s}^{-1} \mathrm{~cm}^{-2}$. It was accompanied by a hardening

\footnotetext{
3 https://www.cosmos.esa.int/web/xmm-newton/xsa
}

of the spectral index from $1.9 \pm 0.7$ to $0.95 \pm 0.30$, that allowed us to register the source with the ART-XC telescope (Fig.2).

The optical counterpart of SRGA J204318.2+443815 ZTF18abjpmzf is a nearly persistent optical source that has been monitored by optical surveys, including the Sloan Digital Sky Survey (SDSS, Alam et al. 2015) DR12, the Panoramic Survey Telescope and Rapid Response System (Pan-STARRS, PS1) DR1 (Flewelling et al. 2020, Waters et al. 2020), and the ZTF DR5.

We also obtained forced photometry on ZTF difference images using the publicly available too ${ }^{4}$ (Masci et al. 2019). For each band, we computed the sum of flux density in the reference image and difference images. The full optical light curve of ZTF18abjpmzf is shown at the bottom panel of Fig. 11.

The optical photometry from the ZTF survey allows one to search for the possible orbital period in the system. From a measured equivalent width of $\mathrm{H}_{\alpha}$ line $E W\left(H_{\alpha}\right)=-17.40 \pm 0.07 \AA$ (see Sec. 6.2) using a relation between $E W$ and the orbital period for BeXRBs (see, e.g. Reig 2011a) we can expect an orbital period to be about 50 days. Otherwise, from the Corbet diagram and known spin period one could estimate the SRGA J204318.2+443815 orbital period to be $\approx 200$ days.

Similarly, we performed forced photometry of the PGIR20fah - NIR counterpart of ZTF18abjpmzf - using the PGIR images based on the method described in De et al. (2020a). The $J$-band light curve is shown in Fig. 1 .

To search for the source orbital period we calculated a generalized Lomb-Scargle periodogram (Scargle 1982) from all available ZTF $r$-filter measurements. We also produced a hundred simulated data sets with similar level of red noise at higher frequencies. No significant peaks are observed in periodogram. Similar analysis was carried out for PGIR data with no prominent peaks found.

${ }^{4}$ See details in http://web.ipac.caltech.edu/staff/fmasci/ ztf/forcedphot.pdf 


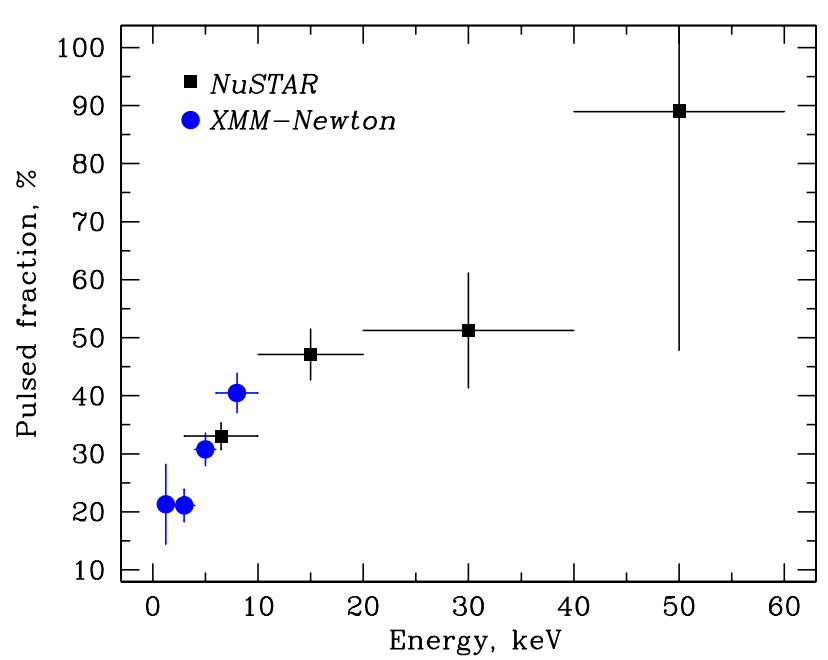

Fig. 5. Pulsed fraction of SRGA J204318.2+443815 as a function of the energy as observed by XMM-Newton (blue circles) and NuSTAR (black squares).

\section{Timing analysis and discovery of pulsations}

Soon after the discovery of the source with ART-XC we triggered a TOO observation with the XMM-Newton observatory in order to study its properties in details. The observation has been performed around 3 weeks after the discovery. As can be seen from Fig. 1 the source was found in the same intensity state. For the timing analysis we applied the barycentric correction. Using standard epoch folding technique we were able to discover very strong pulsations of flux with period $P_{\mathrm{s}}=741.4 \pm 0.3 \mathrm{~s}$ (see Fig. 3). About two weeks after the XMM-Newton observation SRGA J204318.2+443815 was observed with NuSTAR. Using these data we were able to confirm presence of pulsations in the source light curve with period $P_{\mathrm{s}}=741.8 \pm 0.1 \mathrm{~s}$. As can be seen from period values obtained from the XMM-Newton and NUSTAR data, the source does not experience strong spinup or spin-down that is compatible with the stability of its flux. Uncertainties for the pulse period values were determined from the simulated light curves following the procedure described by Boldin et al. (2013).

Using the corresponding spin periods we constructed the pulse profile of SRGA J204318.2+443815 in different energy bands (Fig. 4). The pulse profile shape demonstrates a clear dependence on the energy. At low energies (below several $\mathrm{keV}$ ) it has simple single-peak structure. At higher energies the multiple peaks become more and more prominent. Already in the 10-20 $\mathrm{keV}$ band the profile consists of four evenly distributed over the spin phase peaks with a comparable intensity. At even higher energies the pulse profile is dominated by two main peaks at phases around 0.1 and 0.8 .

A broad-band coverage provided by joint use of the $X M M$ Newton and NUSTAR data allowed us also to study dependence of the pulsed fraction ${ }^{5}$ on the energy. It was found that it is increasing with the energy (Fig. 5p, which is typical behaviour for the majority of XRPs (e.g., Lutovinov \& Tsygankov 2009). Moreover, the increase rate is changing around $10-15 \mathrm{keV}$, that may point to the presence of different physical mechanisms responsible for the observed emission in different energy bands, or presence of some scattering medium affecting low-energy photons.

\footnotetext{
5 defined as $\mathrm{PF}=\left(F_{\max }-F_{\min }\right) /\left(F_{\max }+F_{\min }\right)$, where $F_{\max }$ and $F_{\min }$ are maximum and minimum fluxes in the pulse profile, respectively
}

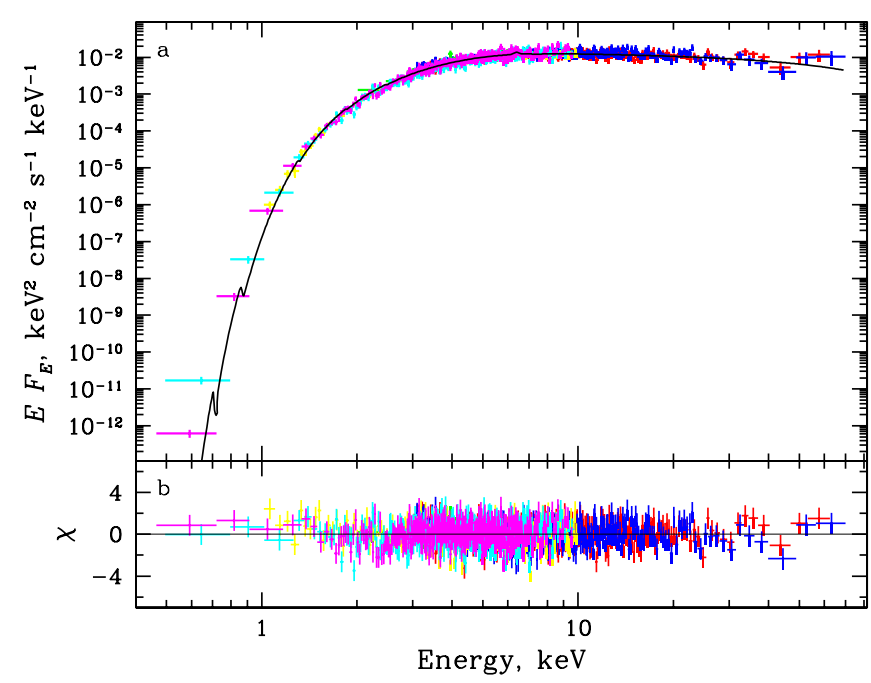

Fig. 6. (a) Energy spectrum of SRGA J204318.2+443815 as measured by Swift/XRT (green points), XMM-Newton (yellow, cyan and magenta points for PN, MOS1 and MOS2 instruments, respectively) and $\mathrm{NuS}$ $T A R$ (blue and red points for FPMA and FPMB modules, respectively). Black line corresponds to the best fit model compTT+GAUss+PHABs. (b) Residuals from the model.

\section{Broad-band X-ray spectrum}

As it follows from the Swift/XRT monitoring and SRG observations, the source flux and spectral parameters evolve only slightly. Therefore we can approximate spectra obtained by XMM-Newton, NuSTAR and Swift/XRT simultaneously.

As it is well known, broad band spectra of X-ray pulsars differ significantly from the spectra of neutron stars with low magnetic field in low mass X-ray binaries and from the spectra of accreting black holes (Syunyaev et al. 1991). The broadband spectrum of SRGA J204318.2+443815 is typical for accreting magnetized neuron stars and demonstrates an exponential cutoff at high energies (Fig.6). Therefore, we fitted it with several continuum models usually used for such objects: a power law with a high energy exponential cutoff (HIGHCUT or CUTOFFPL in the XSPEC package), combination of negative and positive power laws with exponential cutoff and comptonization model coMPTT. Additionally components accounting for the interstellar absorption and fluorescent iron line at $6.4 \mathrm{keV}$ were added as the PHABS model and Gaussian.

The source and background spectra from all three cameras of XMM-Newton (MOS1, MOS2, PN), both the FPMA and FPMB modules of NuSTAR as well as from the Swift/XRT telescope were used for simultaneous fitting. To take into account the uncertainty in the instrument calibrations as well as the lack of full simultaneity of observations, cross-calibration constants between them were included in the spectral model.

All the above mentioned models describe the source spectrum quite adequately, but the compTT model gives the better fit quality $\left(\chi^{2}=1385\right.$ (1279 dof) for CUTOFFPL, 1353 (1278 dof) for POWERLAW*HIGHCUT, 1326 (1278 dof) for coMpTT). The best-fit parameters are summarized in Table 1 .

There are no obvious absorption features connected with cyclotron resonant scattering features in the range $5-50 \mathrm{keV}$, that allowed us to roughly constrain a magnetic field strength of the neutron star as $B<5 \times 10^{11}$ or $B>5 \times 10^{12} \mathrm{G}$.

The conclusion about an absence of additional spectral features was also verified using the phase-resolved spectroscopy, as there are pulsars in which the cyclotron line or its higher har- 
Table 1. Best-fitting results for the SRGA J204318.2+443815 spectrum

\begin{tabular}{lc}
\hline \hline Parameter $^{a}$ & Value \\
\hline$T_{0}, \mathrm{keV}$ & $1.51 \pm 0.03$ \\
$T_{\mathrm{p}}, \mathrm{keV}$ & $24.6_{-7.7}^{+44.0}$ \\
$\tau_{\mathrm{p}}$ & $1.16_{-0.81}^{+0.55}$ \\
$N_{\mathrm{H}}$ & $4.48 \pm 0.11$ \\
$E_{\mathrm{Fe}}, \mathrm{keV}$ & $6.38 \pm 0.05$ \\
$\sigma_{\mathrm{Fe}}, \mathrm{keV}$ & $0.12 \pm 0.06$ \\
$C_{\mathrm{B}}$ & $1.071 \pm 0.015$ \\
$C_{\mathrm{XRT}}$ & $1.083 \pm 0.064$ \\
$C_{\mathrm{MOS} 1}$ & $1.008 \pm 0.015$ \\
$C_{\mathrm{MOS} 2}$ & $0.911 \pm 0.014$ \\
$C_{\mathrm{PN}}$ & $0.914 \pm 0.012$ \\
$F_{\mathrm{X}}, \mathrm{erg} \mathrm{s}^{-1} \mathrm{~cm}^{-2}$ & $5.08 \times 10^{-11}$ \\
$\chi^{2}$ (d.o.f.) & $1325.8(1278)$ \\
\hline
\end{tabular}

${ }^{a}$ Here $T_{\mathrm{p}}, \tau_{\mathrm{p}}$ and $T_{0}$ are the plasma temperature, plasma optical depth, temperature of the seed photons for the coMPTT model. Fluxes are given in the $0.5-100 \mathrm{keV}$ energy range.

monics are appeared only at certain phases of rotation of the neutron star (see, e.g. Molkov et al. 2019, 2021). Particularly, we produced energy spectra of the source in 10 evenly distributed phase bins. Unfortunately, low counting statistics didn't allow us to detect significant variations over the pulse of any spectral parameters of our best fit model.

\section{Optical observations}

\subsection{Optical and Infrared (OIR) Photometry}

ZTF18abjpmzf has been observed by the Two Micron All-Sky Survey (2MASS; Skrutskie et al. 2006), the Wide-field Infrared Survey Explorer (WISE) telescope (Wright et al. 2010), and the Palomar Gattini-IR De et al. (2020d). In the AllWISE catalog (circa 2010, Cutri \& et al. 2013), the source was detected at $W 1=10.868 \pm 0.023, W 2=10.724 \pm 0.021, W 3=10.326 \pm$ 0.107 , and $W 4=8.905 \pm 0.410$ (Vega system). During the post-cryogenic phase from 2014 to 2020, it was also monitored by Near-Earth Object WISE Reactivation Mission (NEOWISE; Mainzer et al. 2014) every 6 months in the $W 1$ and $W 2$ bands. The infrared light curves are presented in Figure 7.

As reported by Yao et al. (2020) and De et al. (2020b), ZTF18abjpmzf exhibits a secular brightening and undulations on timescales of years in the optical and infrared bands. From 2010 to 2020 , the source has clearly brightened by $\Delta g \approx 0.10$, $\Delta r \approx 0.65, \Delta W 1 \approx 0.82$, and $\Delta W 2 \approx 0.98$. ZTF18abjpmzf also exhibits transient fluctuations at the level of $0.1-0.2 \mathrm{mag}$ on the timescale of a few days. These results are also confirmed by RTT150 , revealing long term variations of the object brightness at the time scale of several months (see above). The PGIR $J$-band lightcurve exhibit long term low amplitude variability from the source in addition to a secular brightening of $\approx 0.2$ mag between 2019 November and 2020 May.

Long-term secular variations in brightness and opticalNIR colors in BeXRBs are often associated with the diskloss episodes (see, e.g. Wisniewski et al. 2010, and references therein). During such episodes, the optical source becomes fainter and bluer (Reig \& Fabregat 2015), while during the disk recovery the source is brighten and redden. This is closely reminiscent the observed brightness evolution of SRGA J204318.2+443815 and also agrees well with nondetection of the source in 2011 by XMM-Newton.
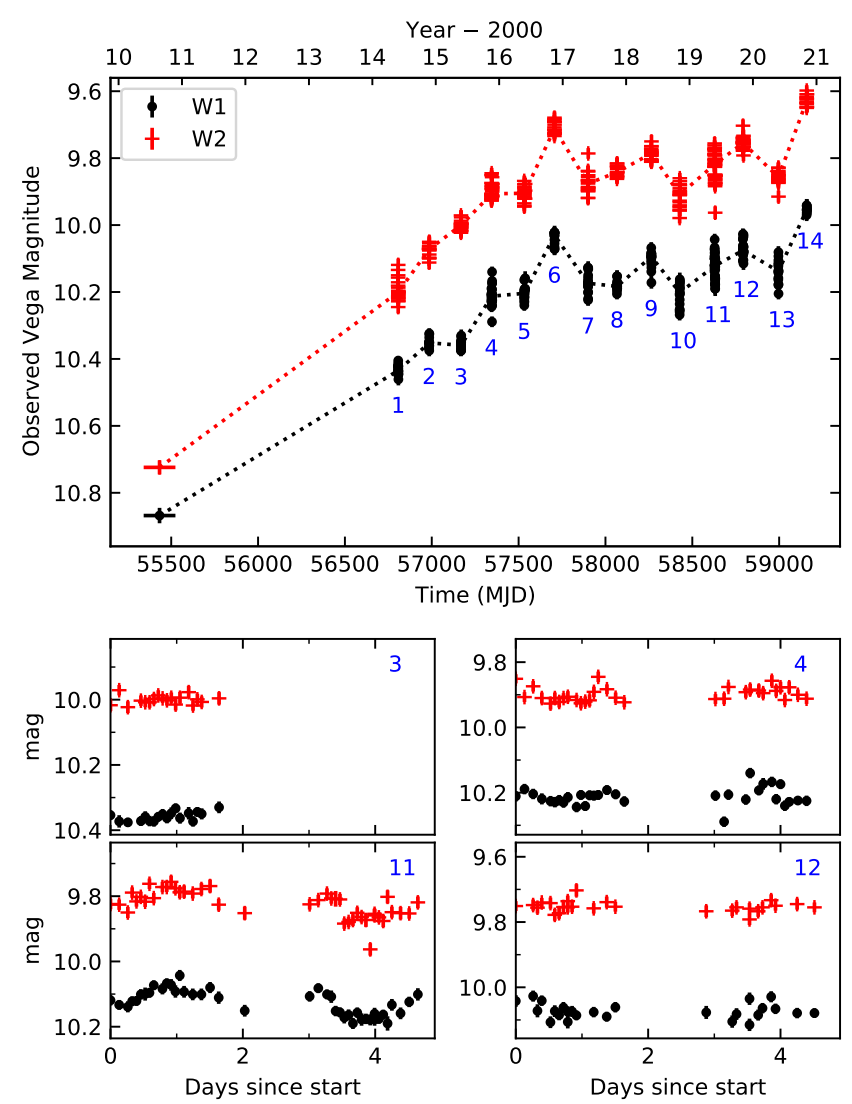

Fig. 7. WISE light curve of ZTF18abjpmzf in $W 1$ and $W 2$ bands. The lower panels show the zoom in of four NEOWISE visits.

\subsection{Optical and Near-Infrared Spectroscopy}

The optical spectrum of ZTF18abjpmzf obtained with RTT-150 on Nov 24, 2020 in frames of the follow-up campaign exhibited a very reddened featureless continuum with only a $\mathrm{H} \alpha$ line in the emission. Two additional RTT-150 spectra with the higher resolution of $\sim 5.5 \AA$ measured on Nov 25, 2020 with the exposure of $3600 \mathrm{~s}$ each allowed us to measure FWHM of the $\mathrm{H}_{\alpha}$ line as $\simeq 13 \AA$ and equivalent width $\mathrm{EW} \simeq-14 \AA$. Taking into account above mentioned instrumental profile, the intrinsic width of the $\mathrm{H}_{\alpha}$ line can be estimated as $\simeq 12 \AA(550 \mathrm{~km} / \mathrm{s})$. This value corresponds to $V_{\sin } i \simeq 275 \mathrm{~km} / \mathrm{s}$, that is agreed with the rotational speed of the equatorial disk around Be star.

Later, in the LRIS and Tspec spectra (Figure 8), we identify strong emission lines of $\mathrm{HI}_{\mathrm{I}}, \mathrm{He}_{\mathrm{I}}$, and Fe II. We also see strong interstellar absorption lines such as $\mathrm{Na}$ I, $\mathrm{K}$ I, and diffuse interstellar bands (DIBs). These indicate a stellar spectrum of Be type (Gray \& Corbally 2009). There is very little flux below $5000 \AA$ due to the high extinction, preventing an accurate spectral type from being assigned. However, the presence of strong emission in the $\mathrm{H}_{\mathrm{I}}$ Paschen series ( $\mathrm{Pa} 7$ to $\left.\mathrm{Pa} 19\right)$ identifies the star as no later than B2 (Andrillat et al. 1988). In the NIR, we observed the $\mathrm{H}_{\mathrm{I}}$ Brackett series (Br7, Br10 to $\left.\mathrm{Br} 24\right)$, as well as $\mathrm{He}_{\mathrm{I}}$ at $1.083 \mu \mathrm{m}, 1.700 \mu \mathrm{m}$, and $2.058 \mu \mathrm{m}$. These emission lines signifies a star earlier than B2.5 (Hanson et al. 1996). The non-detection of $\mathrm{He}_{\mathrm{I}}$ at $2.1116 \mu \mathrm{m}$ and singly-ionized helium is indicative of stars later than O9 (Hanson et al. 1998, 2005). The $\mathrm{H} \alpha$ profile (inset, upper panel of Figure 8) shows a shallow absorption core on top of an emission line with kinematically broadened full-width half-maximum (FWHM) of $460 \mathrm{~km} \mathrm{~s}^{-1}$. This value is slightly lower than ones estimated from RTT-150, 

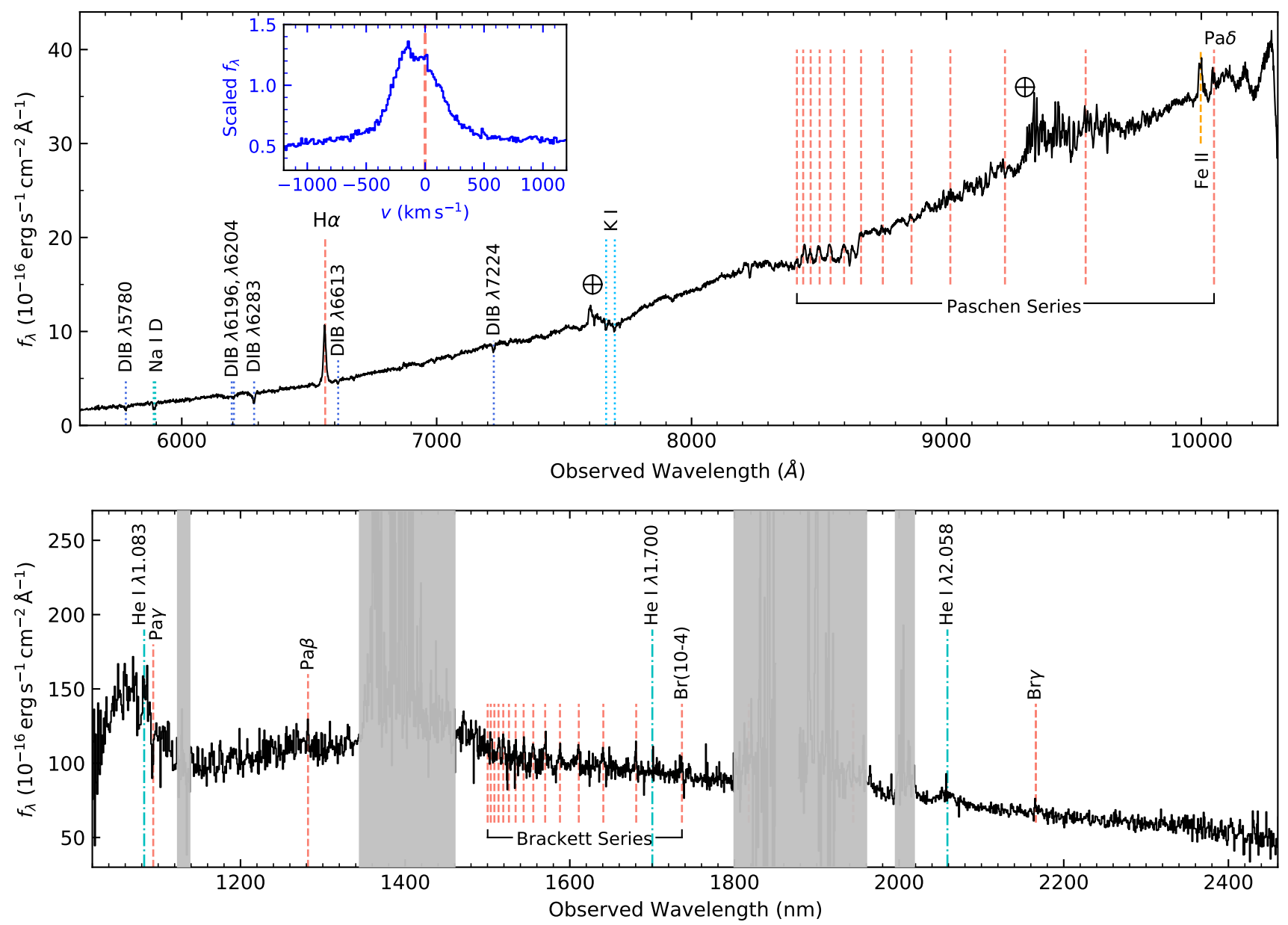

Fig. 8. Upper: Observed LRIS spectrum of ZTF18abjpmzf. The inset shows the H $\alpha$ profile in the ESI spectrum, where the instrumental broadening is $\sigma_{\text {inst }}=16 \mathrm{~km} \mathrm{~s}^{-1}$. Bottom: Observed Tspec spectrum of ZTF18abjpmzf. Wavelength ranges of high atmospheric opacity are masked in grey.
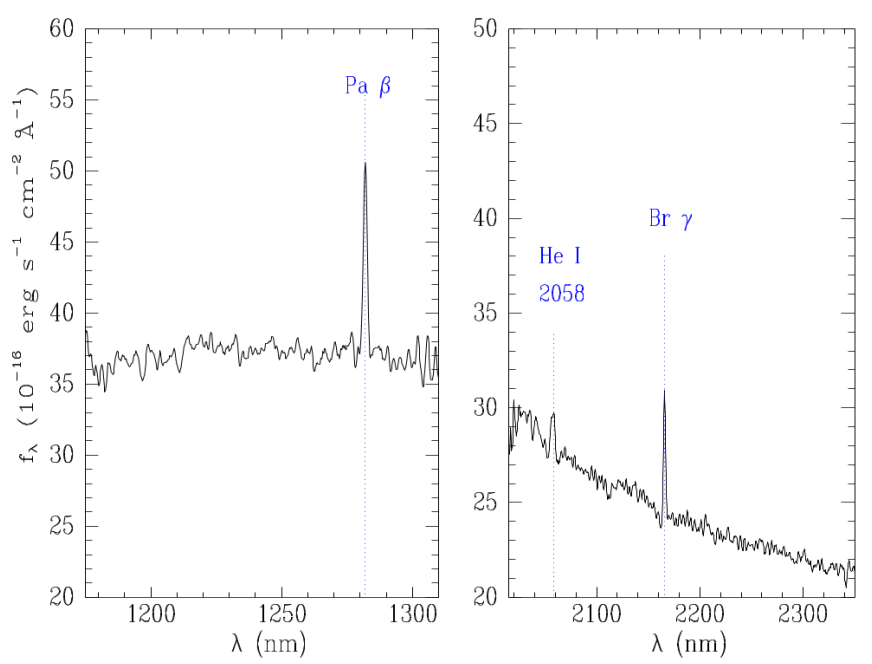

Fig. 9. Parts of the $\mathrm{J}$ and $\mathrm{K}$ band spectra with the prominent emission lines obtained with NOTCam on 2021 Apr 25.

but still compatible with typical rotational speeds of the decretion discs around Be stars and signifies a viewing angle that is not close to edge-on (Hanuschik 1996). Taken together, we conclude that ZTF18abjpmzf contains a star with a spectral type of B0-B2e.
We produced a summed spectrum using the LRIS and ESI data, and measured the equivalent width $(E W)$ of interstellar absorption lines. Using the relations reported by $\mathrm{Mu}-$ nari \& Zwitter (1997) and Yuan \& Liu (2012), the measured $E W\left(\mathrm{~K}_{\mathrm{I}} \lambda 7699\right)=0.47 \pm 0.01, E W(\mathrm{DIB} \lambda 5780)=1.73 \pm 0.06$, and $E W(\mathrm{DIB} \lambda 6283)=3.67 \pm 0.04$ can be converted to $E(B-$ $V)=2.07 \pm 0.03,2.84 \pm 0.10$, and $2.91 \pm 0.03$, respectively. We note that the highly reddened nature of ZTF18abjpmzf requires extrapolation of the nominal relations beyond the calibrated ranges. Therefore, the derived $E(B-V)=2-3$ should be considered as rough estimates.

The NOTCam near-IR spectra taken on 2021 April 25, see Fig. 9. show strong emission lines in $\mathrm{Pa} \beta, \mathrm{Br} \gamma$ and $\mathrm{He}_{\mathrm{I}}$ at $2.058 \mu \mathrm{m}$, the latter quite broad. The continuum flux is lower than that observed with Tspec four months earlier, while the emission lines are stronger, the $\mathrm{Pa} \beta \mathrm{EW}$ is $-6.9 \pm 0.5 \AA$ and the $\mathrm{Br} \gamma \mathrm{EW}$ is $-5.3 \pm 0.5 \AA$ in the NOTCam spectra, which is about the double of that measured four months earlier by Tspec. From the $K$ band acquisition image we used differential photometry with 9 comparison stars to calibrate roughly the flux and found that the target was $0.89 \pm 0.08$ mag brighter than its cataloged 2MASS magnitude.

Now, using the derived constrains on the stellar class of the optical star we can compare observed magnitudes of the IRcounterpart from the 2MASS catalog ${ }^{6}(H=11.904 \pm 0.021$; $K s=11.481 \pm 0.018)$ with the absolute ones of B0-2e stars. Ac-

${ }^{6}$ https://cdsarc.unistra.fr/viz-bin/cat/II/246 
cording to Wegner (2015), the average intrinsic color of such stars is $(H-K s)_{0} \simeq-0.04$. Thus, under the assumption of the standard extinction law (Cardelli et al. 1989), we can estimate the magnitude of the absorption $A_{K s}$ towards the source as $A_{H}-A_{K s}=(H-K s)-(H-K s)_{0}$, which gives $A_{K s} \simeq 0.85$ and $E(B-V) \simeq 2.5$.

Correcting the observed magnitude of the counterpart in the $K s$-filter for the absorption and comparing unabsorbed magnitude with the absolute one, we can estimate (see Karasev et al. 2015 , for details) the distance to the object in the range of 4$7.5 \mathrm{kpc}$, which approximately agrees with the values obtained by Bailer-Jones et al. (2021).

\section{Conclusions}

In this paper we reported a discovery of a new X-ray pulsar SRGA J204318.2+443815/SRGe J204319.0+443820. The source was found by both instruments, ART-XC and eROSITA, on board $S R G$ during second and third all-sky surveys. The follow-up campaign with X-ray and optical observatories allowed to reveal pulsations with the period of $\simeq 742 \mathrm{~s}$, hard $\mathrm{X}$-ray spectrum with the exponential cutoff and a number of emission lines $\left(\mathrm{H}_{\alpha}\right.$, He I, Pashen and Braket series) in the optical and infrared spectra of the companion star. Both these factors as well as the source luminosity of $L_{X} \simeq 4 \times 10^{35} \mathrm{erg} \mathrm{s}^{-1}$, estimated from the X-ray spectrum of the source (Table 11) and Gaia distance $\simeq 8 \mathrm{kpc}$, strongly suggest the BeXRB nature of SRGA J204318.2+443815. Moreover, based on the optical and IR spectral measurements we can restrict a spectral type of the companion star as B0-B2e at an estimated distance of 4-7.5 kpc, that approximately agrees with the Gaia measurements.

Based on the relative stability of the source flux on the time scale of several months, apparently we are dealing with a new member of the subclass of persistent low-luminosity BeXRB systems (see, e.g. Reig 2011b) presumably accreting from the "cold" accretion disk (Tsygankov et al. 2017a b). However historical XMM-Newton data point to the possible variability of the $\mathrm{X}$-ray flux caused by an evolution of the optical companion, that is also seen from the long-term monitoring in optics and IR bands. This joint optical and X-ray variability could be naturally explained in terms of decretion disk loss episode, during which the source was quiescent in X-rays and dimmer in optical-NIR. Later, the replenishment of disk around optical star restarted accretion onto the NS, leading to increase of X-ray flux, and, eventually, to ART-XC detection.

A relatively hard X-ray band of the ART-XC telescope also makes the measured X-ray flux less dependent on the source intrinsic or Galactic absorption and allows the detection of heavily absorbed sources, which could be missed by soft X-ray instruments. Taking into account that the sensitivity of the SRG telescopes are exceeding any previous and currently working surveying instruments, the $S R G$ observatory allow us to unveil the hidden population of faint persistent objects including the population of slowly rotating X-ray pulsars in BeXRB. The potential of $S R G$ to reach this goal is demonstrated by this paper as well as by the discovery of several other new XRPs in the Magellanic Clouds and our Galaxy (see e.g., Haberl et al.|2020, Maitra et al. 2020; Doroshenko et al. 2021).

Acknowledgements. This work is based on observations with Mikhail Pavlinsky ART-XC, and eROSITA X-ray telescopes aboard SRG observatory. The SRG observatory was built by Roskosmos in the interests of the Russian Academy of Sciences represented by its Space Research Institute (IKI) in the framework of the Russian Federal Space Program, with the participation of the Deutsches Zentrum für Luft- und Raumfahrt (DLR). The ART-XC team thanks the Russian Space
Agency, Russian Academy of Sciences and State Corporation Rosatom for the support of the ART-XC telescope design and development. The SRG/eROSITA X-ray telescope was built by a consortium of German Institutes led by MPE, and supported by DLR. The SRG spacecraft was designed, built, launched and is operated by the Lavochkin Association and its subcontractors. The science data are downlinked via the Deep Space Network Antennae in Bear Lakes, Ussurijsk, and Baykonur, funded by Roskosmos. The eROSITA data used in this work were processed using the eSASS software system developed by the German eROSITA consortium and proprietary data reduction and analysis software developed by the Russian eROSITA Consortium. We also would like to thank the XMM-Newton, NuSTAR, NICER and Swift/XRT teams for organising prompt observations. This research has made use of data, software and/or web tools obtained from the High Energy Astrophysics Science Archive Research Center (HEASARC), a service of the Astrophysics Science Division at NASA/GSFC and of the Smithsonian Astrophysical Observatory's High Energy Astrophysics Division. This work made use of data supplied by the UK Swift Science Data Centre at the University of Leicester. Authors (IFB, MRG, RAS) are grateful to TUBITAK, the Space Research Institute, the Kazan Federal University for their partial support in using RTT-150 (the Russian - Turkish 1.5-m telescope in Antalya). Partly based on observations made with the Nordic Optical Telescope, owned in collaboration by the University of Turku and Aarhus University, and operated jointly by Aarhus University, the University of Turku and the University of Oslo, representing Denmark, Finland and Norway, the University of Iceland and Stockholm University at the Observatorio del Roque de los Muchachos, La Palma, Spain, of the Instituto de Astrofisica de Canarias. This work was supported by the Russian Science Foundation (grant 19-12-00423).

\section{References}

Alam, S., Albareti, F. D., Allende Prieto, C., et al. 2015, ApJS, 219, 12

Andrillat, Y., Jaschek, M., \& Jaschek, C. 1988, A\&AS, 72, 129

Arnaud, K. A. 1996, in ASP Conf. Ser., Vol. 101, Astronomical Data Analysis Software and Systems V, ed. G. H. Jacoby \& J. Barnes (San Francisco: Astron. Soc. Pac.), 17-20

Bailer-Jones, C. A. L., Rybizki, J., Fouesneau, M., Demleitner, M., \& Andrae, R. 2021, AJ, 161, 147

Bellm, E. C., Kulkarni, S. R., Graham, M. J., et al. 2019, PASP, 131, 018002

Boldin, P. A., Tsygankov, S. S., \& Lutovinov, A. A. 2013, Astronomy Letters, 39,375

Burrows, D. N., Hill, J. E., Nousek, J. A., et al. 2005, Space Sci. Rev., 120, 165

Cardelli, J. A., Clayton, G. C., \& Mathis, J. S. 1989, ApJ, 345, 245

Cutri, R. M. \& et al. 2013, VizieR Online Data Catalog, II/328

De, K., Ashley, M. C. B., Andreoni, I., et al. 2020a, ApJ, 901, L7

De, K., Hankins, M., Kasliwal, M. M., et al. 2020b, The Astronomer's Telegram, 14234, 1

De, K., Hankins, M. J., Kasliwal, M. M., et al. 2020c, PASP, 132, 025001

De, K., Hankins, M. J., Kasliwal, M. M., et al. 2020d, PASP, 132, 025001

Evans, P. A., Beardmore, A. P., Page, K. L., et al. 2009, MNRAS, 397, 1177

Flewelling, H. A., Magnier, E. A., Chambers, K. C., et al. 2020, ApJS, 251, 7

Gehrels, N., Chincarini, G., Giommi, P., et al. 2004, ApJ, 611, 1005

Graham, M. J., Kulkarni, S. R., Bellm, E. C., et al. 2019, arXiv e-prints [arXiv: 1902.01945]

Gray, R. O. \& Corbally, Christopher, J. 2009, Stellar Spectral Classification

Haberl, F., Maitra, C., Carpano, S., et al. 2020, The Astronomer's Telegram, 13609,1

Hanson, M. M., Conti, P. S., \& Rieke, M. J. 1996, ApJS, 107, 281

Hanson, M. M., Kudritzki, R. P., Kenworthy, M. A., Puls, J., \& Tokunaga, A. T. 2005, ApJS, 161, 154

Hanson, M. M., Rieke, G. H., \& Luhman, K. L. 1998, AJ, 116, 1915

Hanuschik, R. W. 1996, A\&A, 308, 170

Harrison, F. A., Craig, W. W., Christensen, F. E., et al. 2013, ApJ, 770, 103

Herter, T. L., Henderson, C. P., Wilson, J. C., et al. 2008, in Society of PhotoOptical Instrumentation Engineers (SPIE) Conference Series, Vol. 7014, Ground-based and Airborne Instrumentation for Astronomy II, ed. I. S. McLean \& M. M. Casali, 70140X

Karasev, D. I., Tsygankov, S. S., \& Lutovinov, A. A. 2015, Astronomy Letters, 41,394

Lutovinov, A. A. \& Tsygankov, S. S. 2009, Astronomy Letters, 35, 433

Mainzer, A., Bauer, J., Cutri, R. M., et al. 2014, ApJ, 792, 30

Maitra, C., Haberl, F., Carpano, S., et al. 2020, The Astronomer's Telegram, 13610, 1

Masci, F. J., Laher, R. R., Rusholme, B., et al. 2019, PASP, 131, 018003

Mereminskiy, I., Medvedev, P., Lutovinov, A., et al. 2020, The Astronomer's Telegram, 14206, 1

Molkov, S., Doroshenko, V., Lutovinov, A., et al. 2021, arXiv e-prints, arXiv:2106.09514

Molkov, S., Lutovinov, A., Tsygankov, S., Mereminskiy, I., \& Mushtukov, A. 2019, ApJ, 883, L11 
Moore, A. M. \& Kasliwal, M. M. 2019, Nature Astronomy, 3, 109 Munari, U. \& Zwitter, T. 1997, A\&A, 318, 269

Oke, J. B., Cohen, J. G., Carr, M., et al. 1995, PASP, 107, 375

Pavlinsky, M., Tkachenko, A., Levin, V., et al. 2021, A\&A, 650, A42

Predehl, P., Andritschke, R., Arefiev, V., et al. 2021, A\&A, 647, A1

Reig, P. 2011a, Ap\&SS, 332, 1

Reig, P. 2011b, Ap\&SS, 332, 1

Reig, P. \& Fabregat, J. 2015, A\&A, 574, A33

Saxton, R. D., Read, A. M., Esquej, P., et al. 2008, A\&A, 480, 611

Scargle, J. D. 1982, ApJ, 263, 835

Sheinis, A. I., Bolte, M., Epps, H. W., et al. 2002, PASP, 114, 851

Skrutskie, M. F., Cutri, R. M., Stiening, R., et al. 2006, AJ, 131, 1163

Sunyaev, R., Arefiev, V., Babyshkin, V., et al. 2021, arXiv e-prints, arXiv:2104.13267

Syunyaev, R. A., Arefev, V. A., Borozdin, K. N., et al. 1991, Soviet Astronomy Letters, 17, 409

Tsygankov, S. S., Mushtukov, A. A., Suleimanov, V. F., et al. 2017a, A\&A, 608, A17

Tsygankov, S. S., Wijnands, R., Lutovinov, A. A., Degenaar, N., \& Poutanen, J. 2017b, MNRAS, 470, 126

Wachter, K., Leach, R., \& Kellogg, E. 1979, ApJ, 230, 274

Waters, C. Z., Magnier, E. A., Price, P. A., et al. 2020, ApJS, 251, 4

Wegner, W. 2015, Astronomische Nachrichten, 336, 159

Wisniewski, J. P., Draper, Z. H., Bjorkman, K. S., et al. 2010, ApJ, 709, 1306

Wright, E. L., Eisenhardt, P. R. M., Mainzer, A. K., et al. 2010, AJ, 140, 1868

Yao, Y., Kulkarni, S. R., \& Hillenbrand, L. 2020, The Astronomer's Telegram, 14232,1

Yuan, H. B. \& Liu, X. W. 2012, MNRAS, 425, 1763 